\title{
A RESONANT SISO SENSOR BASED ON A COUPLED ARRAY OF MICROELECTROMECHANICAL OSCILLATORS
}

\author{
Barry E. DeMartini ${ }^{1}$, Jeffrey F. Rhoads ${ }^{2}$, Steven W. Shaw ${ }^{2}$, and Kimberly L. Turner ${ }^{1}$ \\ ${ }^{1}$ Department of Mechanical and Environmental Engineering, University of California - Santa Barbara \\ Santa Barbara, California USA \\ ${ }^{2}$ Department of Mechanical Engineering, Michigan State University \\ East Lansing, Michigan USA
}

\begin{abstract}
This work details the preliminary development and testing of a novel, single input - single output (SISO) resonant microsensor, which exploits vibration localization in a coupled-oscillator architecture to allow for the detection of multiple analytes with a single platform. The work includes a brief description of a preliminary sensor design and its operating principles, an outline of a lumped mass analog that proves convenient for analysis and predictive design, and preliminary experimental results, recently obtained from a first-generation device, which verify the sensor's feasibility.
\end{abstract}

\section{INTRODUCTION}

Chemical and biological sensors based on microresonators are considered viable alternatives to modern sensing systems since they consume less power and space than their macroscale counterparts [1-3]. Traditionally, these systems track resonance shifts in essentially single-degree-of-freedom oscillators induced by mass or stiffness changes. As these changes are caused by local bonding, stress stiffening, or a similar chemical-mechanical process, the resonance shifts, in turn, indicate the presence of a given analyte. Existing sensors require the measurement of the response of an individual resonator for the detection of a specific compound, or a class of compounds. Large sensor arrays composed of isolated microresonators can be used to broaden detection capabilities [4], but the addition of the attendant electronics (arising from a larger number of system outputs) adds to the complexity of such sensors.

The present work introduces a sensor design that is intended to couple the inherent benefits of a sensor array with those of a SISO system. The system is realized by coupling an array of simple microbeam resonators with slightly different natural frequencies to a common shuttle mass, which is used for both actuation and sensing (see Fig. 1). The benefit of this architecture is that, due to vibration localization in the set of mistuned beams and their coupling to the shuttle mass, frequency shifts of any of the individual oscillators can be tracked through the response of the shuttle mass [5]. This, in turn, allows for the detection and identification of multiple analytes with a single platform.

The next section provides a brief description of a representative sensor, the formulation of a lumped mass model, and an analysis of the system response. This is followed by a description of the results obtained from a preliminary experimental investigation. The paper concludes with a brief discussion of design and integration issues and a summary of planned work.

\section{THEORY}

Though the sensor described herein can be implemented in a variety of geometrical forms, the translational design depicted in Fig. 1 was selected as the focus of this work. This relatively simple sensor geometry consists of a shuttle mass (SM) that is connected to ground by folded beam supports (S) and excited by interdigitated electrostatic comb drives (CD). Attached to the shuttle mass are four microbeams (M), each of slightly different length to allow for ample separation of the system's natural frequencies.

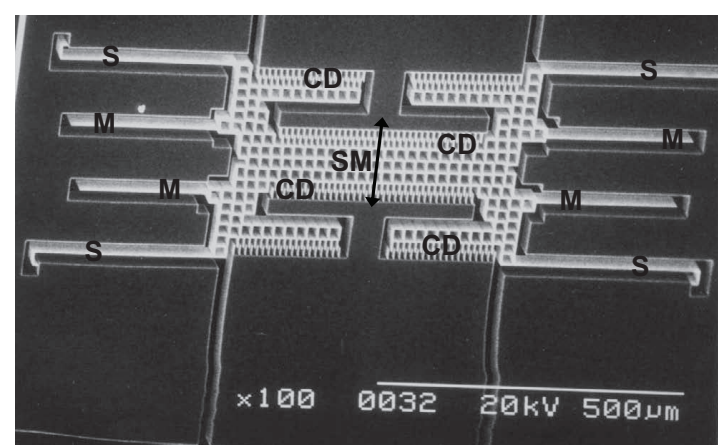

Figure 1. A scanning electron micrograph of the sensor. The device's shuttle mass is labeled SM, the individual microbeams $M$, the electrostatic comb drives $C D$, and the folded beam supports $S$. The dominant direction of motion is indicated by the arrow.

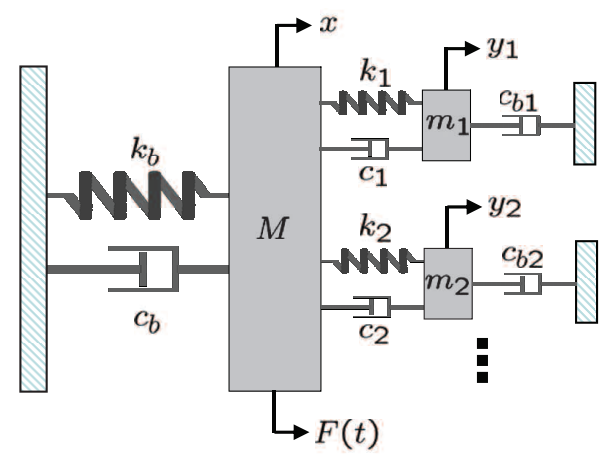

Figure 2. Mass-spring-dashpot analog of the sensor depicted in Fig. 1. Note that the comparatively larger mass $M$ is used to represent the shuttle mass and the smaller masses $m_{i}$ represent the microbeams.

For ease of analysis it proves convenient to represent the device depicted in Fig. 1 as an equivalent mass-spring-dashpot system, as depicted in Fig. 2, where the shuttle mass is represented by mass $M$ and the microbeams by the smaller masses $m_{1}, m_{2}$, etc. From this linear analog the system's equations of motion are easily determined to be

$$
\begin{aligned}
& M \ddot{x}+\sum_{i} m_{i}\left(\ddot{x}+\ddot{z}_{i}\right)+\sum_{i} c_{b i}\left(\dot{x}+\dot{z}_{i}\right)+c_{b} \dot{x}+k_{b} x=F(t) \\
& m_{i}\left(\ddot{x}+\ddot{z}_{i}\right)+c_{b i}\left(\dot{x}+\dot{z}_{i}\right)+c_{i} \dot{z}_{i}+k_{i} z_{i}=0, \quad i=1, \ldots, N
\end{aligned}
$$


where $z_{i}$ is the relative displacement of the $i$ th subsystem given by

$$
z_{i}=y_{i}-x, \quad i=1, \ldots, N
$$

$c_{b}, c_{i}$, and $c_{b i}$ represent equivalent linear damping coefficients arising from various dissipation effects, and $F(t)$ represents the applied electrostatic force generated by the comb drives. Assuming harmonic voltage excitation and ample device thickness, $F(t)$ can be approximated by

$$
F(t)=\frac{\varepsilon_{0} n h V_{A}^{2}}{2 g}(1+\cos 2 \omega t)
$$

where $\varepsilon_{0}$ represents the free space permittivity, $n$ the number of comb fingers, $g$ the gap between adjacent comb fingers, $h$ the device thickness, and $V_{A}$ the amplitude of the applied AC voltage. Nondimensionalizing Eqs. (1) and (2) and redefining by translation the dynamic variable $x$ to remove the explicit appearance of a DC equilibrium shift (see Eq. 4), allows the equations of motion to be compiled into a standard matrix form given by

$$
M X^{\prime \prime}+C X^{\prime}+K X=\Gamma(\tau)
$$

where $X$ represents the compiled state vector, $M$ the effective mass matrix (incorporating inertial coupling terms), $C$ the effective damping matrix (incorporating dissipative coupling terms), $K$ the effective stiffness matrix, and $\Gamma(\tau)$ the effective forcing vector, which is sparse except for the first element. The sensor's response can be determined using this matrix equation through a number of standard techniques (e.g., the impedance approach adopted in [6]).

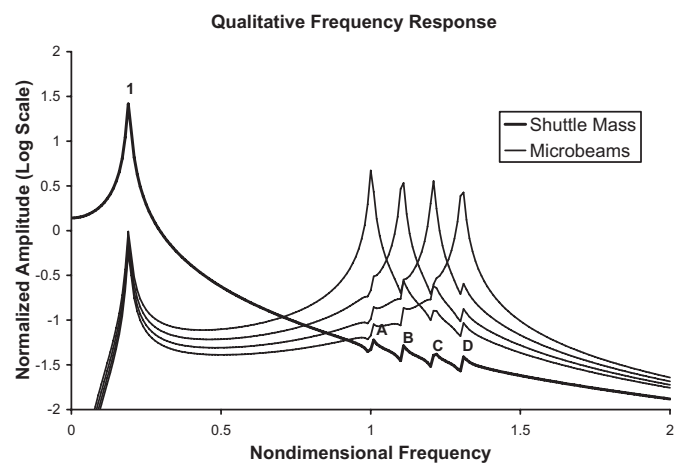

Figure 3. Qualitative frequency response for the sensor. Note that the labeled resonances correspond to the following modes: (1) A translational in-plane mode, (A, B, C, and D) modes where energy is localized in one of the system's microbeams.

A desirable form of the system frequency response, recovered using an approach similar to that outlined in [6], is shown in Fig. 3. As evident, the shuttle mass' response features a dominant lowfrequency resonance, labeled (1), corresponding to a bulk, in-plane translational mode, and four comparatively higher-frequency (and lower-amplitude) resonances, labeled (A), (B), (C), and (D), corresponding to in-plane modes where the system's energy is largely confined to, that is, localized in, a single microbeam (as evident from the relative resonant amplitudes of the microbeams). Sweeping the system's excitation frequency through the region that contains resonances (A), (B), (C), and (D) allows for the detection of resonance shifts, induced by mass and/or stiffness changes in any of the individual microbeams, using solely the measured response of the shuttle mass.
As noted above, the qualitative frequency response shown in Fig. 3 depicts a desirable form of the system's frequency response. This, however, is not the qualitative response that is recovered for arbitrarily selected system parameters. To recover the response shown in Fig. 3, and thus to ensure proper sensor operation, system parameters must be carefully selected. Due to the relative simplicity of the sensor geometry presented herein, the only system parameters pertinent for design are the system inertia ratios (defined as the ratio between a given microbeam's effective modal mass and that of the shuttle), the system frequency ratios (defined as the ratio between the fundamental natural frequency of a given isolated microbeam and the first natural frequency of the shuttle mass), and the amplitude of the AC voltage excitation applied to the electrostatic comb drives. This driving amplitude is used primarily to control the amplitude of the shuttle mass response, so that the resonance peaks of interest are measurable, and the system response remains linear.

Selection of the frequency ratios is used primarily to ensure ample separation between resonances (A), (B), (C), and (D) and all other system resonances, including those related to both in-plane and out-of-plane modes. If these four resonances are placed too close to the resonance corresponding to the bulk, in-plane mode they become saturated and shifts in a given resonance become difficult to detect. Similarly, if the resonances are too close to a resonance corresponding to an out-of-plane mode, the resonant amplitudes decrease and it becomes quite difficult to detect resonance shifts using solely the shuttle mass' amplitude response. There must also be ample separation between each of the four resonances, as placing the resonances too close to one another leads to the elimination of the distinct resonance peaks, and thus undermines detection.

Selection of the inertia ratios is used primarily to manipulate the coupling strength, and thus, is used in conjunction with the frequency ratios, to control the extent of localization in the microbeams [7]. For sensing, sufficient localization is essential for the identification of the source of a resonance shift. Since the microbeams are coupled to the shuttle mass, mass or stiffness changes in a single oscillator induce shifts in all of the coupled system natural frequencies. However, if the response of the microbeams is sufficiently localized, mass or stiffness changes in a single oscillator induce markedly larger shifts in the resonance associated with that particular oscillator. This allows for not only the detection of resonance shifts using the shuttle mass response, but also the identification of the source of the resonance shift (in this case, the specific microbeam that induced it). By providing selective surface chemistry on the microbeams this should allow for rapid, and potentially automated, identification of a target analyte or group of analytes.

\section{EXPERIMENTAL RESULTS}

The device depicted in Fig. 1 was fabricated using a standard SOI process flow. In order to validate the feasibility of the sensor design, mass detection was simulated by comparing the response of the shuttle mass before and after the deposition of a small amount of mass on the shortest (highest frequency) microbeam. The device was placed in partial vacuum and the shuttle mass frequency response was obtained through the use of a laser vibrometer [8]. As shown in Fig. 4, the sensor exhibited a baseline frequency response qualitatively compatible with that predicted in Fig. 3, which was produced using approximate system parameters. In particular, the experimental frequency response displayed the predicted low-frequency resonance (1) corresponding to the bulk, in-plane mode, two resonances $(2,3)$ corresponding to out-of- 
plane modes, and four predicted higher-frequency resonances (A, $\mathrm{B}, \mathrm{C}$, and D) corresponding to the localized microbeam modes. The localized nature of these vibration modes was verified using a stroboscopic in-plane measurement system and video imaging. While the resonances corresponding to the out-of-plane modes (2, 3) were not predicted using the lumped mass model developed in the previous section, which describes only in-plane modes, it is worth noting that these resonances had been predicted by modal simulations performed using a commercial finite element package. Additionally, the separation between resonance (3) and (A) was smaller than desired, and may render resonance (A) as not useful for sensing (These issues can be easily addressed in future designs.).

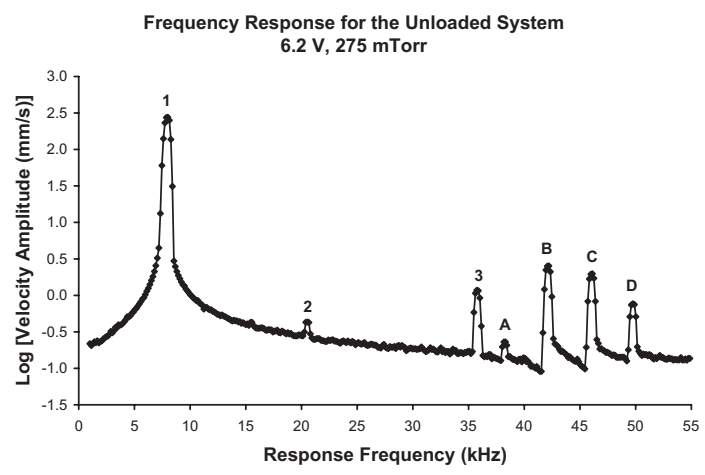

Figure 4. Experimentally-obtained frequency response for the sensor actuated with a $6.2 \mathrm{~V}$ AC signal in 275 mTorr pressure. Note that the labeled resonances correspond to the following modes: (1) Bulk in-plane mode, (2, 3) out-of-plane modes, (A, B, $C$, and $D)$ modes where energy is localized in one of the system's microbeams.

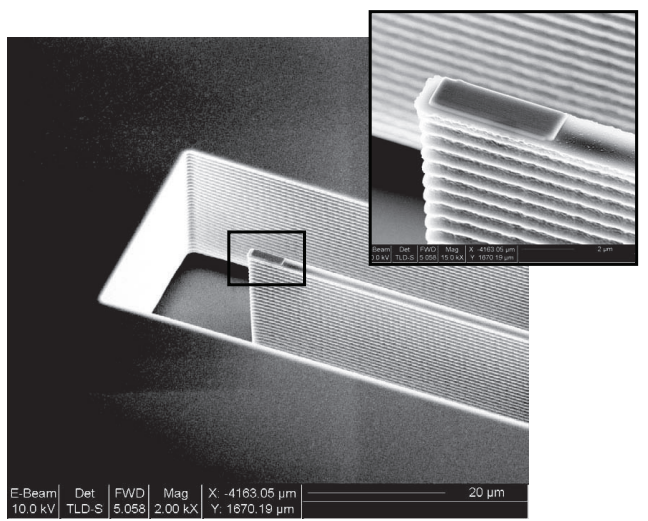

Figure 5. A scanning electron micrograph of the platinum patch added to the shortest microbeam. The inset, which was used for measurement purposes, shows a closer view. The patch is approximately $1.57 \times 5.10 \times 0.22 \mu \mathrm{m}$ in size and has a mass of about $38 \mathrm{pg}$.

Once the baseline frequency response was obtained, a Focused Ion Beam (FIB) was used to deposit a $1.57 \times 5.10 \times 0.22$ $\mu \mathrm{m}$ platinum patch on the desired microbeam, as shown in Fig. 5 . This resulted in an approximate mass change, computed by volume, of $38 \mathrm{pg}$. The frequency response diagrams obtained for the sensor shuttle mass with and without the platinum mass addition are shown in Figs. 6-8. As predicted, the mass addition induced shifts in all of the system's resonances. However, due to vibration localization, the shift in resonance (D) was about 40 times that of the other peaks. Specifically, resonance (D) shifted by approximately $124 \mathrm{~Hz}$ as compared to the next largest resonance shift, which occurred in resonance (C), which was approximately $3 \mathrm{~Hz}$ (see Figs. 6-8). This indicates an experimental mass resolution of approximately $3.3 \mathrm{~Hz} / \mathrm{pg}$ a value comparable to alternative multi-analyte sensor designs [4].

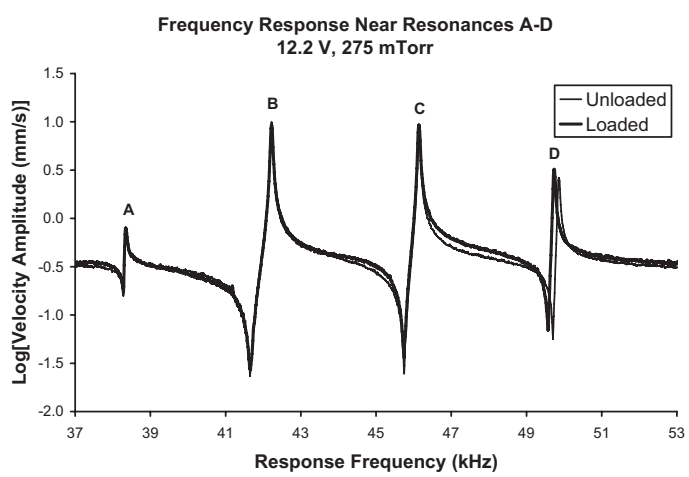

Figure 6. Experimentally-obtained frequency response for the sensor actuated with a $12.2 \mathrm{~V}$ AC signal in 275 mTorr pressure. Note that the added-mass loading introduces resonance shifts, the largest of which occurs in resonance (D), which corresponds to the localized mode of the corresponding beam. Here, and in Figs. 7 and 8, individual data points have been removed for clarity.

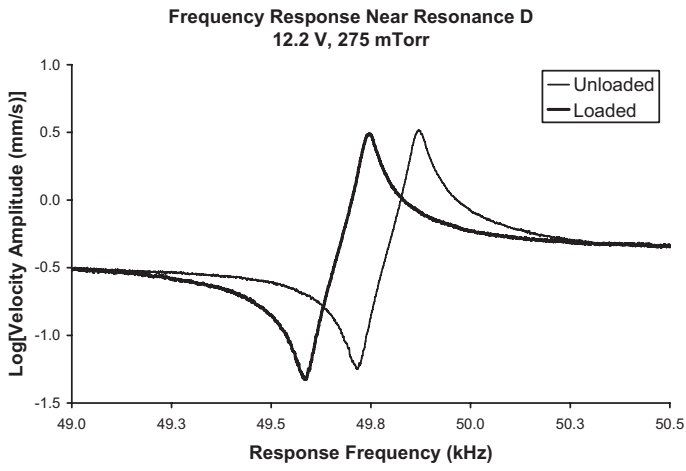

Figure 7. Experimentally-obtained frequency response near resonance (D) for the sensor actuated with a $12.2 \mathrm{~V}$ AC signal in 275 mTorr pressure. Note that mass loading introduces a resonance shift of $124 \mathrm{~Hz}$.

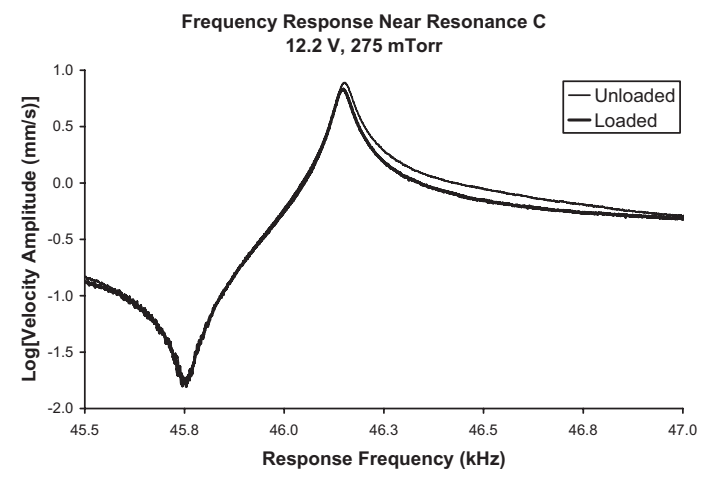

Figure 8. Experimentally-obtained frequency response near resonance (C) for the sensor actuated with a $12.2 \mathrm{~V}$ AC signal in 275 mTorr pressure. Note that mass loading introduces a resonance shift of about $3 \mathrm{~Hz}$. 


\section{DESIGN AND INTEGRATION}

While the preceding sections include brief descriptions of some of the issues that must be considered in the course of sensor development, it is important to briefly discuss a few others relating to both design and integration. Foremost amongst these are sensor metrics, in particular, mass resolution. The sensor described herein was designed with proof of concept and not maximum metrics in mind. A mass resolution of approximately $3.3 \mathrm{~Hz} / \mathrm{pg}$ was measured, and while this value compares favorably to other reported values for multi-analyte resonant sensors [4], it is less than those reported for sensors based on isolated microresonators [2]. Device scaling will help to improve mass resolution, but physical sensing limits and the fact that shifts in the shuttle mass frequency response are measured, rather than shifts in isolated microbeam resonances, dictate that the maximum attainable mass resolution will generally be less than those of its uncoupled counterparts $[\mathbf{9 , 1 0}]$.

Another important issue to be considered in the course of development is the method of response measurement. In this preliminary study measurement was a secondary concern, as it had been predetermined that a laser vibrometer would be employed to obtain the system frequency response. The electrostatic comb banks that were included in the original device design could be used in conjunction with an external or integrated circuit for measurement purposes, but the effective capacitance change the drives yield near resonance (approximately $0.4 \mathrm{fF}$ ) is not of sufficient magnitude, even if increased slightly by drive reconfiguration, to be measured with a high degree of accuracy. As such, future designs would likely benefit from the inclusion of piezoelectric or magnetomotive drive and/or sensing mechanisms, as the voltage signals induced from material strain or emf effects should be easier to measure using standard external or integrated circuitry.

Another issue that must be considered in the course of design and development is the actual sensor environment. As detailed in the preceding section, all of the experimental results described herein were obtained for a device in partial vacuum. This was done since the resonances were not sufficiently prominent at 1 atm, due to the small $\mathrm{Q}$ values associated with the resonance peaks of interest. While a low pressure environment may be suitable for some sensing activities, the vast majority will require that the device operate in ambient pressure conditions. Geometrical reconfiguration of the in-plane device and device scaling may well be sufficient to allow for this, as fluid pumping between the microbeams and the sidewalls of the substrate is believed to be a large source of dissipation in the present design. Alternative geometries (e.g. in-plane torsional geometries) and phase response analysis, two issues currently being explored, may also aid sensing at $1 \mathrm{~atm}$.

\section{CONCLUSION}

This work details a novel, single input - single output (SISO) resonant microsensor that exploits vibration localization in a coupled array of microbeams to allow for the detection of multiple analytes with a single device. It was shown that a linear lumped mass model for the sensor proves sufficient for both analysis and predictive design. A preliminary device design based on these ideas was fabricated and tested using a simulated added mass. The device was experimentally determined to have a mass resolution of approximately $3.3 \mathrm{~Hz} / \mathrm{pg}$, a value comparable to those reported for other multi-analyte resonant microsensors, yet less than those values reported for sensors based on isolated microresonators.
While the results of this preliminary study are believed to be a positive indication of the sensor's feasibility, a number of issues still must be considered, most notably, improving the sensor mass resolution, introducing a more robust measurement method, integrating the sensor with a circuit capable of measuring shifts in each of the shuttle mass resonances, and determining configurations that result in higher $\mathrm{Q}$ values. These issues, along with a number of others pertinent to development, are currently under consideration.

\section{ACKNOWLEDGEMENTS}

This work was graciously supported by the National Science Foundation under contract NSF-ECS-0428916.

\section{REFERENCES}

[1] J. R. Vig, R. L. Filler, and Y. Kim, "Chemical Sensor Based on Quartz Microresonators", Journal of Microelectromechanical Systems, 5, 138 (1996).

[2] B. Ilic, D. Czaplewski, M. Zalalutdinov, H. G. Craighead, P. Neuzil, C. Campagnolo, C. Batt, "Single Cell Detection with Micromechanical Oscillators", Journal of Vacuum Science and Technology B, 19, 2825 (2001).

[3] T. Thundat, P. I. Oden, and R. J. Warmack, "Microcantilever Sensors", Microscale Thermophysical Engineering, 1, 185 (1997).

[4] H. P. Lang, M. Hegner, and C. Gerber, "Cantilever Array Sensors", Materials Today, 8, 30 (2005).

[5] A. Alsuwaiyan and S. W. Shaw, "Localization of Free Vibration Modes in Systems of Nearly Identical Vibration Absorbers", Journal of Sound and Vibration, 228, 703 (1999).

[6] L. Meirovitch, Elements of Vibration Analysis (1986).

[7] C. Pierre and E. H. Dowell, "Localization of Vibrations by Structural Irregularity", Journal of Sound and Vibration, 114, 549 (1987).

[8] K. L. Turner, P. G. Hartwell, and N. C. MacDonald, "MultiDimensional MEMS Motion Characterization Using Laser Vibrometry", Proceedings of Transducers '99: The 10th International Conference on Solid State Sensors and Actuators, Sendai, Japan, 6/7-10/99 (1999), pp. 1144-1147.

[9] G. Abadal, Z. J. Davis, B. Helbo, X. Borrise, R. Ruiz, A. Boisen, F. Campabadal, J. Esteve, E. Figueras, F. Perez-Murano, and N. Barniol, "Electromechanical Model of a Resonating NanoCantilever-Based Sensor for High-Resolution and High-Sensitivity Mass Detection”, Nanotechnology, 12, 100 (2001).

[10] K. L. Ekinci, Y. T. Tang, and M. L. Roukes, "Ultimate Limits to Inertial Mass Sensing Based Upon Nanoelectromechanical Systems", Journal of Applied Physics, 95, 2682 (2004). 